\title{
Responding Empathically: A Question of Heart, not a Question of Skin
}

\section{Patrícia Oliveira-Silva \& Óscar F. Gonçalves}

\section{Applied Psychophysiology} and Biofeedback In association with the Association for Applied Psychophysiology and Biofeedback

ISSN 1090-0586

Appl Psychophysiol Biofeedback DOI $10.1007 /$ s10484-011-9161-2
Volume 33, Number 4

December 2008

$33(4) 181-250(2008)$ ISSN 1090-0586

Applied Psychophysiologog and Biofeedback

Springer

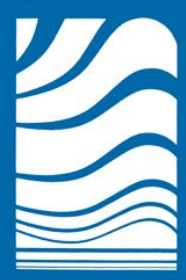


Your article is protected by copyright and all rights are held exclusively by Springer Science+Business Media, LLC. This e-offprint is for personal use only and shall not be selfarchived in electronic repositories. If you wish to self-archive your work, please use the accepted author's version for posting to your own website or your institution's repository. You may further deposit the accepted author's version on a funder's repository at a funder's request, provided it is not made publicly available until 12 months after publication. 


\title{
Responding Empathically: A Question of Heart, not a Question of Skin
}

\author{
Patrícia Oliveira-Silva • Óscar F. Gonçalves
}

(C) Springer Science+Business Media, LLC 2011

\begin{abstract}
Empathy entails the capacities to resonate with another person's emotions, understand his/her thoughts and feelings, separate our own thoughts and emotions from those of the observed and responding with the appropriate prosocial and helpful behavior. While there is abundant research on the neurobiological mechanisms of some components of empathy (e.g., emotional contagion), few studies have considered the neurobiological mechanisms underlying the empathic response. The present study explores psychophysiological correlates (skin conductance level and the interbeat interval) as a function of the empathic response while participants watch and respond to actors portraying emotionally laden vignettes. Forty undergraduate psychology students were each presented with 40 emotional vignettes of positive or negative valence and asked to choose among three different empathic responses while their electrodermal and cardiac responses were measured. Overall, the study shows that higher levels of additive empathy are associated with increased cardiac activity (i.e., decreased Interbeat Interval) but not electrodermal activity.
\end{abstract}

Keywords Empathy - Electrodermal activity - Interbeat interval

\section{Introduction}

Empathy is defined as the ability to apprehend and respond to the emotional state of another person (Batson et al.

P. Oliveira-Silva · Ó. F. Gonçalves ( $₫)$

Neuropsychophysiology Lab, CIPsi, School of Psychology,

University of Minho, Campus de Gualtar, 4710-057 Braga,

Portugal

e-mail: goncalves@psi.uminho.pt
1987). Empathy represents one of the most fundamental human capacities and has been the object of study in both social (Eisenberg 2002; Hoffman 2000) and clinical domains (Feshbach 1997; Olsen 2001).

Traditionally, two main components of empathy have been addressed in the psychological literature: emotional and cognitive. While the emotional component refers to the process of emotional contagion (i.e., sharing the emotional processes of another person), the cognitive component addresses our capacity to understand the inner world of another person (i.e., to take the perspective of someone else). De Waal (2008) claims that these two components refer to different evolutionary stages. He suggests a "Russian doll model of empathy" in which the inner layer refers to the most basic process of emotional contagion, which evolves into a second layer of sympathetic concern, which, in turn, evolves into a final layer of perspectivetaking. This final layer requires a separation between the emotions and thoughts of the observer and the emotions and thoughts portrayed by the observed, which corresponds to what Decety and Grèzes (2006) refer as the self-agency dimension of empathy.

In sum, empathy entails the capacity to resonate with another person's emotions, understand his/her thoughts and feelings and, finally, separate our own thoughts and emotions from those of the observed.

Absent from all these models is a fourth component of empathy: the empathic response. That is, how empathic understanding is translated into prosocial behavior such as friendship, altruism, and help. De Vignemont and Singer (2006) refer to this fourth component as the social role of empathy, contrasting it with the epistemological role of empathy (i.e., empathic understanding). This socially responsive component of empathy has been the object of intense study in psychotherapy, at least since 
Rogers (1957) stated it is one of the core necessities and sufficiently enough for clinical change (cf. Bohart and Greenberg 1997).

Despite the increased interest in empathic processes seen in clinical literature, at least two issues related to the empathic response remain to be addressed: (1) the relationship between the empathic response and physiological arousal and (2) the identification of the most sensitive physiological markers of empathic response. While there is abundant research on the neurobiological mechanisms of emotional understanding (cf. Decety and Jackson 2006; Singer and Lamm 2009), few studies have considered the neurobiological mechanisms underlying empathic response.

Several studies using autonomic measures (Eisenberg and Strayer 1987; Eisenberg et al. 1991) have shown that children experiencing increased physiological arousal exhibit decreased prosocial behavior when faced with a person in need. Furthermore, results from dyadic relationship studies employing infant-mother (Cohn and Tronick 1988), patient-clinician (Marci and Orr 2006), or marital dyads (Levenson and Ruef 1992) have suggested that similarities in psychophysiological responses may improve social closeness. For example, physiological concordance between clients and therapists has been associated with higher evaluation of therapists' empathy by the clients (Marci et al. 2007). Analogously, Levenson and Ruef (1992) have shown that accurate emotional rating of one's spouse is associated with low cardiovascular arousal. Taken together, these studies provide initial evidence that some physiological markers may be associated with the empathic response.

Since Rogers' (1957) early formulation of the empathic response, several authors have discussed the need to differentiate various levels of empathy. Carkhuff (1969), for example, has suggested the existence of several levels of empathic response ranging from those that are subtractive and dismissive of another person's experience, to those that reflect a deep understanding of a person's feelings. In-between those extremes we also observe responses that are, in essence, interchangeable, showing that the responder is in-tune with the feelings of another person. More recently, Ivey et al. (2010) have suggested that emphatic responses could be clustered into three different levels: subtractive, interchangeable, and additive.

We believe that these different levels of empathy may be associated with different cognitive processes that could be investigated by use of specific psychophysiological markers. For example, the subtractive levels of empathy seem to be unresponsive to the content of another person's feelings and, as such, no specific psychophysiological reactions would be expected. In contrast, for intermediate levels of empathy, when an individual is responding at an interchangeable level, some form of emotional contagion may occur that will likely be associated with increased autonomic arousal, and therefore, may more accurately be quantified using a skin conductance measure. Finally, high levels of additive empathy require an individual to both connect to, and maintain some type of cognitive distance in order to develop a theory of another's mind. Therefore, additive levels of empathy would seem to imply higher levels of cognitive processing which are more sensitive to measures of cardiac response.

The present study explores the relationship between physiological arousal and the empathic response. More specifically, the main goal of this study is to analyze the relationship between psychophysiological measures (skin conductance level and the interbeat interval) and the empathic response while participants watch and respond to actors portraying emotionally laden vignettes. We hypothesize that: (1) interchangeable level of empathy, being more dependent on emotional contagion, may be preferentially associated with skin conductance responses; (2) additive levels of empathy, which are more dependant on theory-of-mind mechanisms, would be more sensitive to measures of cardiac response; and (3) given the unresponsiveness of subtractive levels of empathy, no specific associations would be expected between subtractive levels of empathy and psychophysiological measures (e.g., skin conductance, cardiac-response measures).

\section{Methods}

\section{Participants}

Forty undergraduate psychology students (15 males, and 25 females; mean $=24.74, \mathrm{SD}=1.19$, range $=17-52$ years) participated in this study in exchange for course credits. None of the participants reported history of neurological or psychiatric problems nor of alcohol or drugrelated abuse. All data from a male participant and half the data from a female participant (20 vignettes) were excluded from the statistical tests due to low data quality. All participants gave written informed consent prior to any procedure. The study was previously approved by the local ethics committee.

\section{Apparatus and Materials}

\section{Emotional Vignettes}

Forty videotaped vignettes of actors portraying emotionally laden situations of positive or negative valence were developed. Each vignette was 27 -s long and performed by professional actors (two males and two females). Negativevalence vignettes focused on emotions such as disappointment, illness, anger, fear, embarrassment or loss; 
while positive vignettes included situations of enthusiasm, satisfaction, delight, personal achievement, dedication and love. An initial pool of 60 vignettes was object of validation by ninety-three psychology majors in terms of emotional valence (i.e., positive or negative), retaining 40 from those with $100 \%$ agreement among raters.

\section{Empathic Response Task}

For each vignette three possible verbal responses with different levels of empathy were provided: (1) empathically subtractive (i.e., a response out of the focus of the expressed emotion); (2) empathically interchangeable (i.e., a response mirroring the expressed emotion); and, (3) empathically additive (i.e., a response helping with the clarification of the expressed emotion). These categories were based on Carkhuff's empathy scale (Carkhuff 1969).

Empathic responses were validated by two experienced clinical psychologists, retaining only those that were correctly classified in terms of the different empathy levels by the two experts. Initially, no agreement was found in the classification of empathic responses in 14 vignettes. Responses to those vignettes were rewritten and submitted to additional expert classification. Still 8 vignettes were not correctly classified and were object of the same processes (rewriting and expert validation). At the end of this process, responses to two vignettes were not yet meeting agreement and were redone in order to reach, finally, a correct classification in terms of the different empathic levels by both experts.

\section{Skin Conductance Level}

The skin's electrical conductivity depends on the activation of the eccrine glands, which are regulated exclusively by the sympathetic branch of the autonomic nervous system, without parasympathetic input or neuro-hormonal influence (Fowles 1986). Due to this direct relationship between sympathetic activation and electrodermal activity, skin conductance has been one of the most used electrophysiological indices of physiological arousal and has been employed in diverse conditions and levels of overall autonomic activity. Skin conductance measures were obtained while participants were watching and responding to the emotional vignettes. As recommended by Fowles et al. (1981), a GSR100C module (BIOPAC MP150) employing a $.5 \mathrm{~V}$ constant voltage technique was used. The silver-silver chloride electrodes (contact area: $6 \mathrm{~mm}$; cavity to accommodate electrode gel: $1.6 \mathrm{~mm}$ ) were nonpolarizable; they were filled with hypotonic gel (.05 molar) and attached to the skin with double-sided adhesive collars. The conductance was recorded from the distal phalanges of the second and third fingers of the nondominant hand. The electrode cables were attached with tape to minimize any mechanical motion. The estimate of the SCL during the task was calculated as a percentage increase or decrease from the original baseline level.

\section{Cardiac Response}

Cardiac activity reflects sympathetic as well as parasympathetic activation. The cardiovascular system is a sophisticated physiological system subject to central, peripheral and humoral influences, and it is highly sensitive to a variety of neurobehavioral processes and psychological or physiological conditions. Electrocardiograms (ECG) continually reflect both rapid and slow fluctuations due to physiological reactivity. Although IBI and heart rate are similar measures, IBI was chosen as the cardiac measure due to its linear relationship with autonomic changes (Stern et al. 2001). The IBI was calculated from ECGs collected with a modified Lead II placement (below each clavicle and the last left rib), using disposable pregelled $\mathrm{Ag}-\mathrm{AgCl}$ electrodes and $7 \%$ chloride gel. Before the placement of the electrodes, the skin was cleaned with alcohol and dried with cotton. A $35 \mathrm{~Hz}$ high-pass filter was used to minimize muscle artifacts. The IBI was computed in milliseconds using the medians of the interval between consecutive R-spikes (Stern et al. 2001) and then converted to a percentage change from the baseline.

\section{Procedures}

After the acquisition of informed consent, participants were asked to clean their hands with neutral soap and water and then to sit in a comfortable position in front of a PC display in a temperature-controlled recording room. The task was performed on a Pentium I computer, using Presentation software (Neurobehavioral Systems, Inc., San Francisco, CA) to control the presentation of the stimuli and register the participants' responses. During the instructions, a simulation trial was performed to ascertain whether participants understood the task. Participants were instructed that the task would last around $45 \mathrm{~min}$ and consist of 40 blocks, each with three distinct phases: (1) watching the video, (2) mentally formulating a response and (3) choosing one among three response options indicating different levels of empathy. While participants were performing the empathic task, physiological indices were recorded with the non-invasive and high-sensitivity modules ECG100C and GSR100C from the Biopac MP150 data acquisition system (Biopac System, Inc., Santa Barbara, USA).

The vignettes were pseudo-randomized, counterbalancing the sequence as well as the actors and emotional valence. As shown in Fig. 1, after the fixation point, an instruction screen was displayed for $3 \mathrm{~s}$ instructing the 


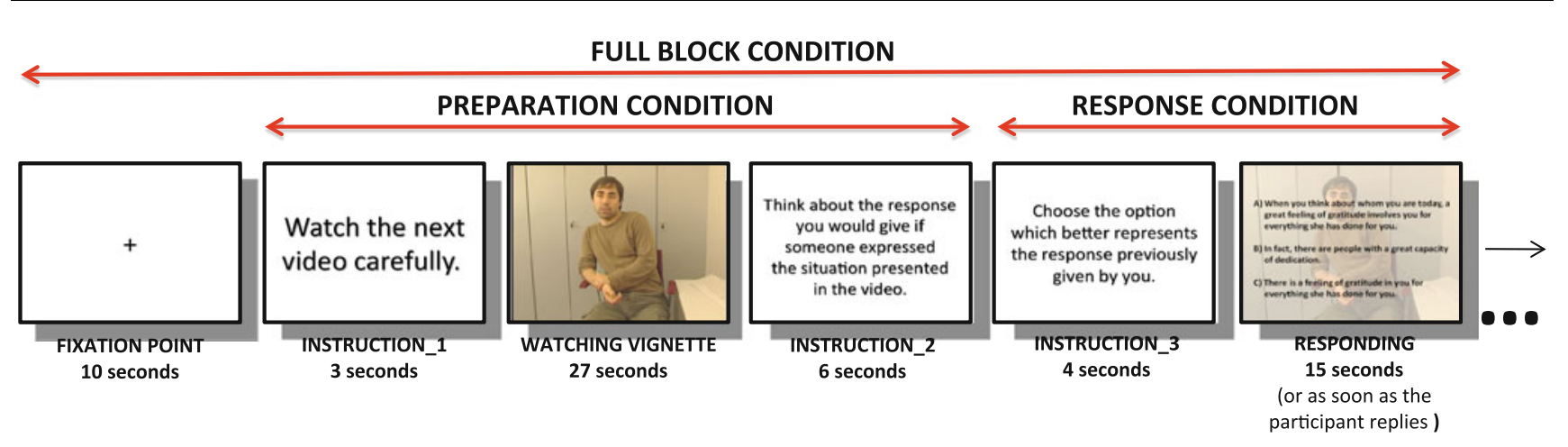

Fig. 1 Illustration of how perform the empathic task

participant to watch the vignette. Next, the vignette was presented for $27 \mathrm{~s}$, followed by a second instruction screen lasting $10 \mathrm{~s}$. During the presentation of the second instruction screen, the participant mentally rehearsed a response. Finally, a third instruction screen was presented asking the participant to choose, via keyboard, one among the three possible responses. After the participant's response, a new block was introduced. A 5-min period before the introduction of the emotional vignettes was used to establish baseline skin conductance and cardiac response.

Three time conditions were selected for psychophysiological analysis. The Full Block condition spanned the entire block from the onset of the vignette presentation to the selection of the empathic response. The Preparation condition included the period during which the subject was instructed to observe and mentally elaborating a response to the vignette. Finally, the Response condition was limited to the period of choosing one of the empathic responses provided.

\section{Results}

The main goal of this study was to analyze the psychophysiological correlates of arousal (skin conductance level and the interbeat interval) as a function of empathic response level while participants attended, were preparing and responded to emotionally laden vignettes.

Non-parametric procedures were used for the analysis of the variables because they were not normally distributed. The non-parametric Kruskal-Wallis test was performed to examine the relationship between the physiological indices and the level of empathic response to the different emotional vignettes. Post-hoc Mann-Whitney tests for pairwise comparisons of independent samples were computed when significant differences were found with the Kruskal-Wallis test. Bonferroni corrections for multiple comparisons were made, and the resulting $p$-value of .016 was set as our threshold for statistical significance.
As depicted in Table 1 and Fig. 2, no significant differences in SCL were found at the different empathic levels (subtractive, interchangeable, additive) either for the full block condition (Kruskal-Wallis test; $H=.018, d f=2$, $p=$ n.s.), the preparation condition (Kruskal-Wallis test; $H=.031, d f=2, p=$ n.s.), or the response condition (Kruskal-Wallis test; $H=.041, d f=2, p=$ n.s.). Analysis of standardized and non-standardized skin conductance scores provided identical results. However, significant differences in the IBI at all three empathic responses levels were found for the full block condition (Kruskal-Wallis test; $H=8.36, d f=2, p=.015)$, the preparation condition (Kruskal-Wallis test; $H=8.92, d f=2, p=.012$ ), and the response condition (Kruskal-Wallis test; $H=10.4$, $d f=2, p=.006$ ). Post-hoc pairwise comparison confirmed that additive empathic responses were significantly associated with lower IBIs compared with the interchangeable responses for all the conditions (see Table 1, Fig. 3). Additionally, during the response condition, additive empathic responses were associated with significantly lower IBIs than subtractive empathic responses but only for the response condition (see Table 1, Fig. 3).

\section{Discussion}

The current study explored the pattern of physiological responses, as revealed by SCL and IBI, associated with different levels of empathic response.

As initially hypothesized, the additive level (i.e., responses with the highest level of empathy) are associated with a significant decrease in IBI, representing heart rate acceleration, when compared to the interchangeable level (for all the conditions) and subtractive levels (only for the response condition). That is, at the moment of responding at higher levels of empathy, participants showed a significant increase in heart rate activity as evidenced by a decrease in the IBI. This finding suggests that cardiovascular activity appears to be sensitive to the level of empathic response to emotionally laden vignettes, and in 
Table 1 Kruskal-Wallis test used to analyze the difference among the empathic levels in three different time conditions of the task, regarding to the SCL (Skin conductance level) and IBI (Interbeat interval), and the post hoc Mann-Whitney tests for pairwise comparisons

\begin{tabular}{|c|c|c|}
\hline \multicolumn{3}{|l|}{ Kruskal-Wallis test } \\
\hline Moment & $H(d f)$ & $p$ value \\
\hline \multicolumn{3}{|l|}{ Skin conductance level (SCL) } \\
\hline Full block & $.018(2)$ & n.s. \\
\hline Preparation & $.031(2)$ & n.s. \\
\hline Response & $.041(2)$ & n.s. \\
\hline \multicolumn{3}{|l|}{ Interbeat interval (IBI) } \\
\hline Full block & $8.36(2)$ & $.015^{*}$ \\
\hline Preparation & $8.92(2)$ & $.012 *$ \\
\hline Response & $10.4(2)$ & $.006^{*}$ \\
\hline \multicolumn{3}{|c|}{ Post hoc Mann-Whitney test (concerned to Interbeat Interval-IBI) } \\
\hline Comparison & U de Mann-Whitney $(Z)$ & $p$ value \\
\hline Subtractive versus interchangeable (Block) & $52,210.5(-.46)$ & n.s. \\
\hline Subtractive versus interchangeable (Preparation) & $52,519.0(-.34)$ & n.s. \\
\hline Subtractive versus interchangeable (Response) & $51,769.5(-.64)$ & n.s. \\
\hline Interchangeable versus additive (Block) & $200,500.0(-2.83)$ & $.005 * *$ \\
\hline Interchangeable versus additive (Preparation) & $199,895.0(-2.92)$ & $.004 * *$ \\
\hline Interchangeable versus additive (Response) & $201,692.0(-2.66)$ & $.008 * *$ \\
\hline Subtractive versus additive (Block) & $77,398.0(-1.38)$ & n.s. \\
\hline Subtractive versus additive (Preparation) & $77,027.0(-1.48)$ & n.s. \\
\hline Subtractive versus additive (Response) & $73,303.0(-2.47)$ & $.013 * *$ \\
\hline
\end{tabular}

* Statistically significant results $(p<.05)$

** Statistical significance evaluate according the Bonferroni correction $(p<.016)$
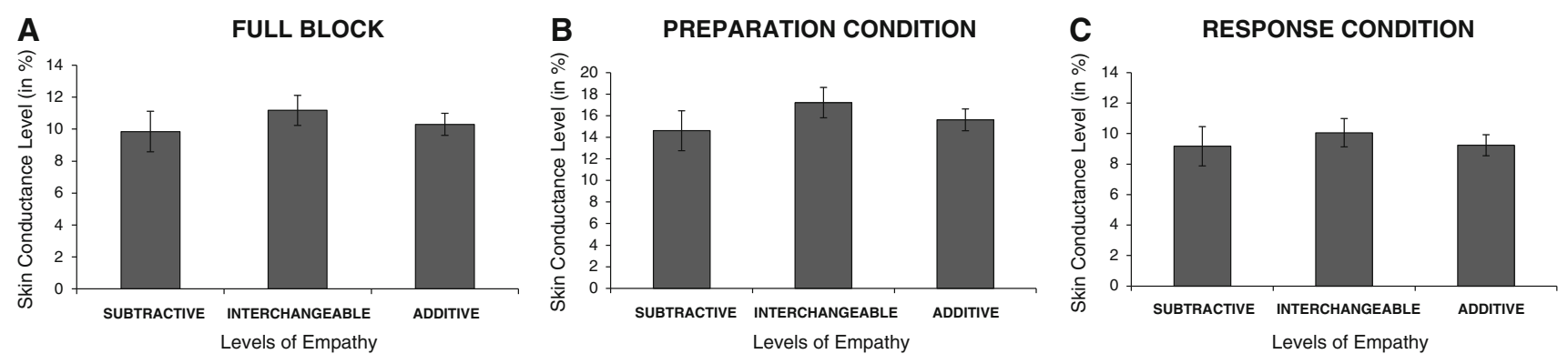

Fig. 2 Percentage of change of the skin conductance level (SCL) during the empathic task (error bars represent the standard error)
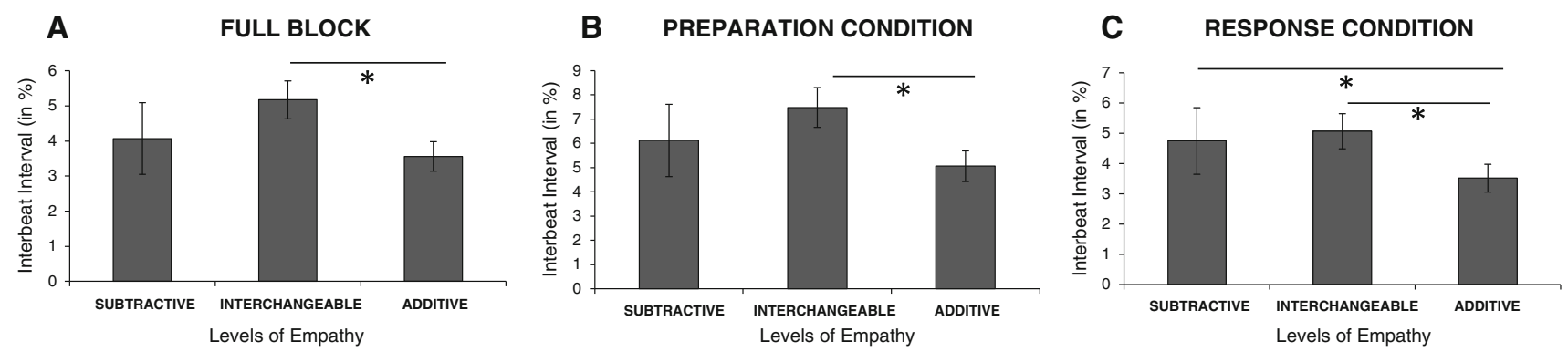

Fig. 3 Percentage of change of the interbeat interval (IBI) during the empathic task (error bars represent the standard error) 
turn, this may represent a important autonomic biomarker of additive empathic response.

On the contrary, and contrary to our initial hypothesis, no significant tonic differences in SCL were found between the three levels of empathic response. SCL is one of the components of electrodermal activity, and provides a reliable measure of emotional arousal, tending to increase in high arousal situations (Greenwald et al. 1989). SCL is probably more closely related to intense sympathetic activation making it a better marker of personal distress reactions than empathic response (Eisenberg and Fabes 1990). It is possible that the short length of our vignettes, along with the fact that the emotions portrayed were of mild emotional intensity, limited their efficacy in triggering processes of emotional contagion that would be tackled by the SCL response.

Our data showed that additive empathic responses are accompanied by an increase in heart rate (i.e., decrease in IBI values) without a corresponding increase in electrodermal activity (i.e., SCL). These results may seem contradictory because increased sympathetic activity would expect to be shown in both physiological responses. To explain this apparently incongruous pattern of SCL and IBI measures, we must take into consideration that multiple biological subsystems are involved in the regulation of cardiovascular activity, namely the central nervous system, the humoral system and reciprocal activation of the sympathetic and parasympathetic branches of the autonomic nervous system (Cacioppo et al. 2007). These influences make the cardiovascular system highly sensitive to many neurobehavioral processes (attention, memory, motor activity, brain reward, etc.). The cardiac response to a specific emotional stimulus may accelerate or decelerate depending on the context, the participant or the method used to evoke emotion (Vrana and Lang 1990). Additionally, the cardiovascular response seems to be more sensitive to experimental task demands (Lang et al. 1990) than to physiological arousal. This specific sensitivity of the cardiac response makes it a good candidate for a biomarker of more complex and multidimensional processes such as the additive empathic response.

As stated above, the human empathic response is a very complex process involving, at minimum, emotional contagion, cognitive understanding and perspective-taking. A good empathic response implies that the individual is able to move beyond emotional arousal, and in doing so, the same individual will not only be able to tune in with the emotions of others but take their perspective and respond appropriately with prosocial behavior. Given that cardiac reactivity is a good measurement of synergistic and reciprocal sympatho-parasympathetic balance and/or the reflex of neurobehavioral processes, it may be a much better biomarker of the additive empathic response compared to SCL.
A full understanding of the role of the cardiovascular system as a biomarker of complex social-emotional responses, such as empathy, would require the inclusion of additional cardiac measurements that could better distinguish more subtle patterns of physiological reactivity (e.g., heart rate variability, peripheral resistance and blood flow volume). Future research should also study the effect of more extended high arousal vignettes in order to further test the hypothesis if interchangeable level of empathy, being more dependent on emotional contagion, are preferentially associated with skin conductance responses.

Acknowledgments The authors are greatly indebted to the BIAL Foundation by the grant "The Neuropsychophysiological Basis of Empathy: The role of neuroendocrine; autonomic and central nervous system variables (89/08)" that supported this research.

\section{References}

Batson, C. D., Fultz, J., \& Schoenrade, P. A. (1987). Distress and empathy: Two qualitatively distinct vicarious emotions with different motivational consequences. Journal of Personality, 55, $19-40$.

Bohart, A. C., \& Greenberg, L. S. (Eds.). (1997). Empathy reconsidered. Washington, DC: American Psychological Association.

Cacioppo, J. T., Tassinary, L. G., \& Berntson, G. G. (2007). Handbook of psychophysiology (3rd ed.). New York: Cambridge University Press.

Carkhuff, R. R. (1969). Helping and human relations: Vol. II practice and research. New York: Holt, Rinehart and Winston.

Cohn, J. F., \& Tronick, E. Z. (1988). Mother-infant face-to-face interaction: Influence is bidirectional and unrelated to periodic cycles in either partner's behavior. Developmental Psychology, 24(3), 386-392.

De Vignemont, F., \& Singer, T. (2006). The empathic brain: How, when and why? Trends in Cognitive Science, 10(10), 435-441.

De Waal, F. B. M. (2008). Putting the altruism back into altruism: The evolution of empathy. Annual Review of Psychology, 59, 279-300.

Decety, J., \& Grèzes, J. (2006). The power of simulation: Imagining one's own and other's behavior. Brain Research, 1079, 4-14.

Decety, J., \& Jackson, P. L. (2006). A social neuroscience perspective on empathy. Current Directions in Psychological Science, 15, $54-58$.

Eisenberg, N. (2002). Empathy-related emotional responses, altruism, and their socialization. In R. J. Davidson \& A. Harrington (Eds.), Visions of compassion: Western scientists and Tibetan Buddhists examine human nature (pp. 131-164). London: Oxford University Press.

Eisenberg, N., \& Fabes, R. A. (1990). Empathy: Conceptualization, measurement, and relation to prosocial behavior. Motivation and Emotion, 14, 131-149.

Eisenberg, N., Fabes, R. A., Schaller, M., Miller, P., Carlo, G., Poulin, R., et al. (1991). Personality and socialization correlates of vicarious emotional responding. Journal of Personality and Social Psychology, 61, 459-470.

Eisenberg, N., \& Strayer, J. (1987). Empathy and its development. Cambridge: Cambridge University Press.

Feshbach, N. D. (1997). Empathy: The formative years-Implications for clinical practice. In A. C. Bohart \& L. S. Greenberg (Eds.), Empathy reconsidered: New directions in psychotherapy (pp. 33-59). Baltimore: United Book Press. 
Fowles, D. C. (1986). The eccrine system and electrodermal activity. In M. G. H. Coles, E. Donchin, \& S. W. Porges (Eds.), Psychophysiology: Systems, processes, and applications (pp. 51-96). New York: Guilford Press.

Fowles, D. C., Christie, M. J., Edelberg, R., Grings, W. W., Lykken, D. T., \& Venables, P. H. (1981). Committee report: Publication recommendations for electrodermal measurements. Psychophysiology, 18, 232-239.

Greenwald, M. K., Cook, E. W., \& Lang, P. J. (1989). Affective judgment and psychophysiological response: Dimensional covariation in the evaluation of pictorial stimuli. Journal of Psychophysiology, 3, 51-64.

Hoffman, M. L. (2000). Empathy and moral development: Implications for caring and justice. Cambridge: University Press.

Ivey, A. E., Ivey, M. B., \& Zalaquett, C. P. (2010). Intentional interviewing and counseling. Belmont, CA: Brooks/Cole.

Lang, P. J., Bradley, M. M., \& Cuthbert, B. N. (1990). Emotion, attention, and the startle reflex. Psychological Review, 97, 377-398.

Levenson, R. W., \& Ruef, A. M. (1992). Empathy: A physiological substrate. Journal of Personality and Social Psychology, 63(2), 234-246.
Marci, C. D., Ham, J., Moran, E., \& Orr, S. P. (2007). Physiologic correlates of perceived therapist empathy and social-emotional process during psychotherapy. Journal of Nervous and Mental Disease, 195, 103-111.

Marci, C. D., \& Orr, S. P. (2006). The effect of emotional distance on psychophysiologic concordance and perceived empathy between patient and interviewer. Applied Psychophysiology and Biofeedback, 31(2), 115-128.

Olsen, D. P. (2001). Empathetic maturity: Theory of moral point of view in clinical relations. Advances in Nursing Science, 24, $36-46$.

Rogers, C. (1957). The necessary and sufficient conditions of therapeutic personality change. Journal of Consulting Psychology, 21, 95-103.

Singer, T., \& Lamm, C. (2009). The social neuroscience of empathy. Annals of the New York Academy of Science, 1156, 81-96.

Stern, R. M., Ray, W. J., \& Quigley, K. S. (2001). Psychophysiological recording (2nd ed.). Oxford, England: Oxford University Press.

Vrana, S. R., \& Lang, P. J. (1990). Fear imagery and the startle probe reflex. Journal of Abnormal Psychology, 99, 181-189. 